\title{
An Interdisciplinary Approach for the Assessment and Implementation of Resilient and Flexible Water Supply Infrastructure under Changing or Instable Conditions
}

\author{
Dr.-Ing. D.I. Mag. Wolfgang K. Walter ${ }^{1}$ and Univ.-Prof. Dr.-Ing. F. W. Günthert ${ }^{2}$ \\ 'DVGW German Technical and Scientific Association for Gas and Water, Germany. \\ ${ }^{2}$ Institute of Hydro Sciences, Universität der Bundeswehr Munich / University of the Federal Armed Forces of \\ Germany at Munich, Germany
}

\begin{abstract}
In case of demographic changes or emergencies pipeline bound infrastructure like gas or water is specifically challenged. Then, the advantages of decentralized infrastructure should be considered: Minimization of asset bound funds; flexibility; resilience; response capacity. A research project at the Federal Armed Forces University together with the Brazilian utility company COPASA, the process engineering company Grünbeck and the ICT company Phönix focused on: A procedure for successful decentralized water infrastructure implementation; a new operation scheme; related pilot tests. To describe the local situation the Open System of Boundaries is created comprising 13 interdisciplinary groups and 68 subgroups. To describe water supply systems the Open System of Attributes is created comprising 15 groups and 64 subgroups. An Attributes Profile which fits into the Boundaries Profile makes a resilient decentralized application more likely. For Minas Gerais, Brazil, i.e. for instable conditions the Boundaries Profile and the Attributes Profile of a decentralized SCADA equipped water treatment plant are compiled. Results of on-site tests are discussed. Recommendations for decentralized water supply under instable conditions are given. The application of standardized SCADA equipped treatment plants is suggested with remote supervision from one control centre.
\end{abstract}

Keywords: Resilience, Decentralised Water Supply, Open System, SCADA

\section{INTRODUCTION}

Today, hard infrastructure and especially grid and pipeline bound infrastructure like electricity, gas, water, wastewater and also traffic are facing numerous challenges on many different levels globally. With regard to water supply three cases can be identified where classical centralized pipeline bound infrastructure systems are specifically challenged:

- Urban or rural connection deprived regions in an instable environment,

- Situations when existing infrastructure has to adapt to upwards or downwards demographic changes,

- The case of emergencies.

Amongst others these cases are commonly characterized by fast or sudden changes in the overall physical and intangible environment, by a future which is hard to predict, by the need for a fast response and resilience or by many interfaces with other infrastructure systems - i.e. by situations unsuitable for inflexible centralized pipeline bound infrastructure systems. 
The water transportation and distribution network of centralized systems often accounts for more than $50 \%$ of the hardware costs with an average service life of up to 50 years. These are long-time bound funds which on the one hand make adaptations in the system difficult and which on the other could be saved by decentralized systems. The advantages of the latter are:

- Minimization of asset bound funds,

- Cost effectiveness,

- Flexibility in management,

- Reliability under varying conditions,

- Resilience and response capacity to sudden changes in the environment,

- Possibility of accelerated implementation,

- According to necessity simple as well as complex technologies possible,

- Because of usually short transport distances, water is delivered in adequate quality to the consumer.

Therefore, in the three cases mentioned above the application of more decentralized infrastructure systems seems promising. This goes especially for connection deprived rural regions in Newly Industrialized Countries NICs.

\section{MACRO-LEVEL SITUATION IN NEWLY INDUSTRIALIZED COUNTRIES NICS}

There exists no universal definition of NICs which was the reason to follow Goldman Sachs papers in selecting BRICS, i.e. Brazil, Russia, India, China and South Africa and Next-Eleven countries, i.e. Bangladesh, Egypt, Indonesia, Iran, Mexico, Nigeria, Pakistan, Philippines, South Korea, Turkey and Vietnam for the assessments.

The analysis of the contribution of their agricultural, industrial and service sector to their national GDP and their water withdrawal distribution between the agricultural, industrial and municipal sector shows that the two are reverse. There is a trend of an increase of the third GDP sector at the cost of the first one. However, by far the largest water withdrawal is dedicated to the first sector which contributes to the GDP little or least.

The urban and rural population distributions do not show a common pattern. BRICS and $\mathrm{N}-11$ countries with a large third sector have a higher urbanization rate in general. Most analyzed countries' annual urbanization rate is superior to the respective population growth rate. The coverage ratios of improved water sources and improved sanitation facilities following WHO definitions are higher in urban areas than they are in rural ones. In rural areas they might even deteriorate which leads to drawbacks like the practice of open defecation in some countries. 


\section{RESEARCH PROJECT}

When an existing central infrastructure system needs to be assessed regarding e.g. its capacity of adaptability or when a hard infrastructure system is to be implemented for the first time two procedures are of upmost importance in order to define the most appropriate and suitable decentralized configuration:

- The precise and multidisciplinary description of the local situation and of the overall boundaries,

- The precise and multidisciplinary assessment of the existing infrastructure system / of the system which is about to be implemented with regard to the local situation.

Both procedures reflect the interactions between hard and soft aspects of infrastructure. However, until now no comprehensive instruments existed in order to carry out the two steps. Deficits in the knowledge about the local situation and about the attributes of infrastructure systems induced numerous infrastructure implementation failures, especially under instable or changing conditions.

A research project at the Universität der Bundeswehr München / University of the Federal Armed Forces of Germany at Munich together with the Brazilian utility company COPASA, the process engineering company Grünbeck and the ICT company Phönix Contact focused on these open questions mainly via the following central research assumptions':

1. The key to a successful application of decentralized water supply is the selection of appropriate systems. Crucial is the knowledge about the local situation and the precise system description.

2. Decentralized water treatment incorporating advanced technology can be applied under instable conditions when the technological aspects are embedded into the socio-economical-cultural ones.

Following the three stage approach of description, systematization and experimentation ${ }^{2}$ studies were carried out in several NICs and DCs. The research region for the exemplary assessment of a modular, SCADA equipped membrane-based decentralized water treatment plant, short pilot plant, was the Brazilian state of Minas Gerais (look figure 1). Qualitative and quantitative methods were applied for the analysis of the empirical data material.

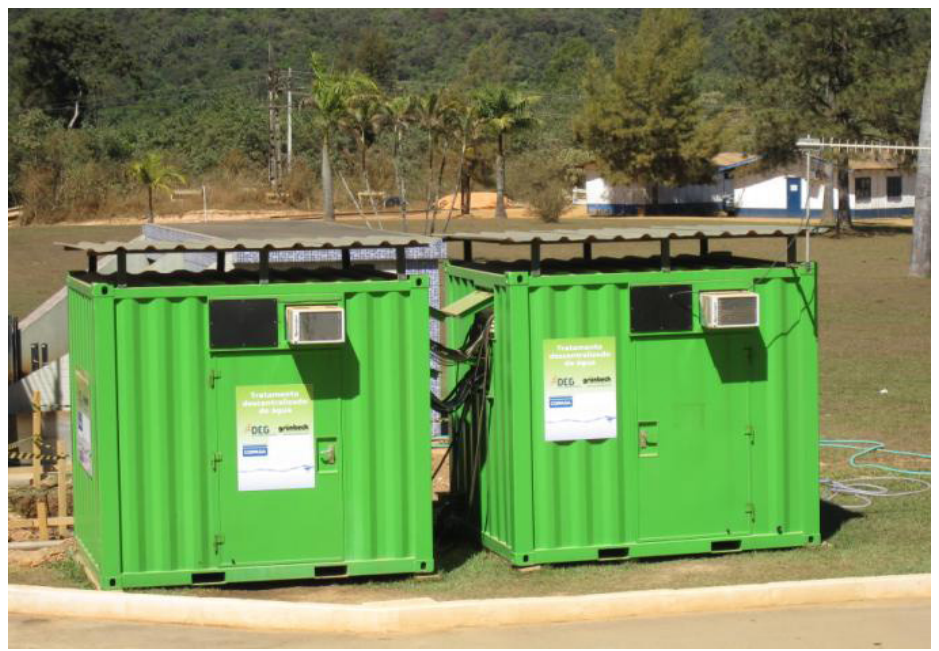

\section{Figure 1: Decentralized water treatment pilot plant in Minas Gerais, Brazil}

Picture: Wolfgang K. Walter

Stemming from the project experience the "Open System of Boundaries for Decentralized Water Supply" is created in order to describe the local situation (look figure 2). It comprises 13 groups of boundaries, e.g. geographical factors, state of local sanitary engineering, macro- and microeconomics, social and cultural factors, etc., including 68 subboundaries.

To describe the properties of water treatment and supply systems the "Open System of Attributes of Decentralized

1 Walter, W.K. Recommendations for Small Water Supply Systems in Newly Industrialized Countries on the Example of Assessments in the State of Minas Gerais, Brazil. Aachen: Shaker (2013).

2 Windelband, W. Präludien - Aufsätze und Reden zur Philosophie und ihrer Geschichte, 2. Band. Tübingen: J.C.B. Mohr, Paul Siebeck (1915). 
Water Supply Systems" is created (look figure 3). It comprises 15 groups of attributes, e.g. system type, process engineering, transportability, social aspects, macro- and microeconomics, etc., including 64 sub-attributes.

For a specific case the "Open System of Boundaries for Decentralized Water Supply" can be employed to describe the local situation. As a result, the local situation is labelled by a distinctive "Boundaries Profile". The "Open System of Attributes of Decentralized Water Supply Systems" is employed to describe water treatment technology in the planning stage or infrastructure which has already been implemented. In both cases, water supply systems get a distinctive "Attributes Profile". An "Attributes Profile" which fits into the "Boundaries Profile" makes a successful, sustainable, resilient and flexible decentralized system application more likely.

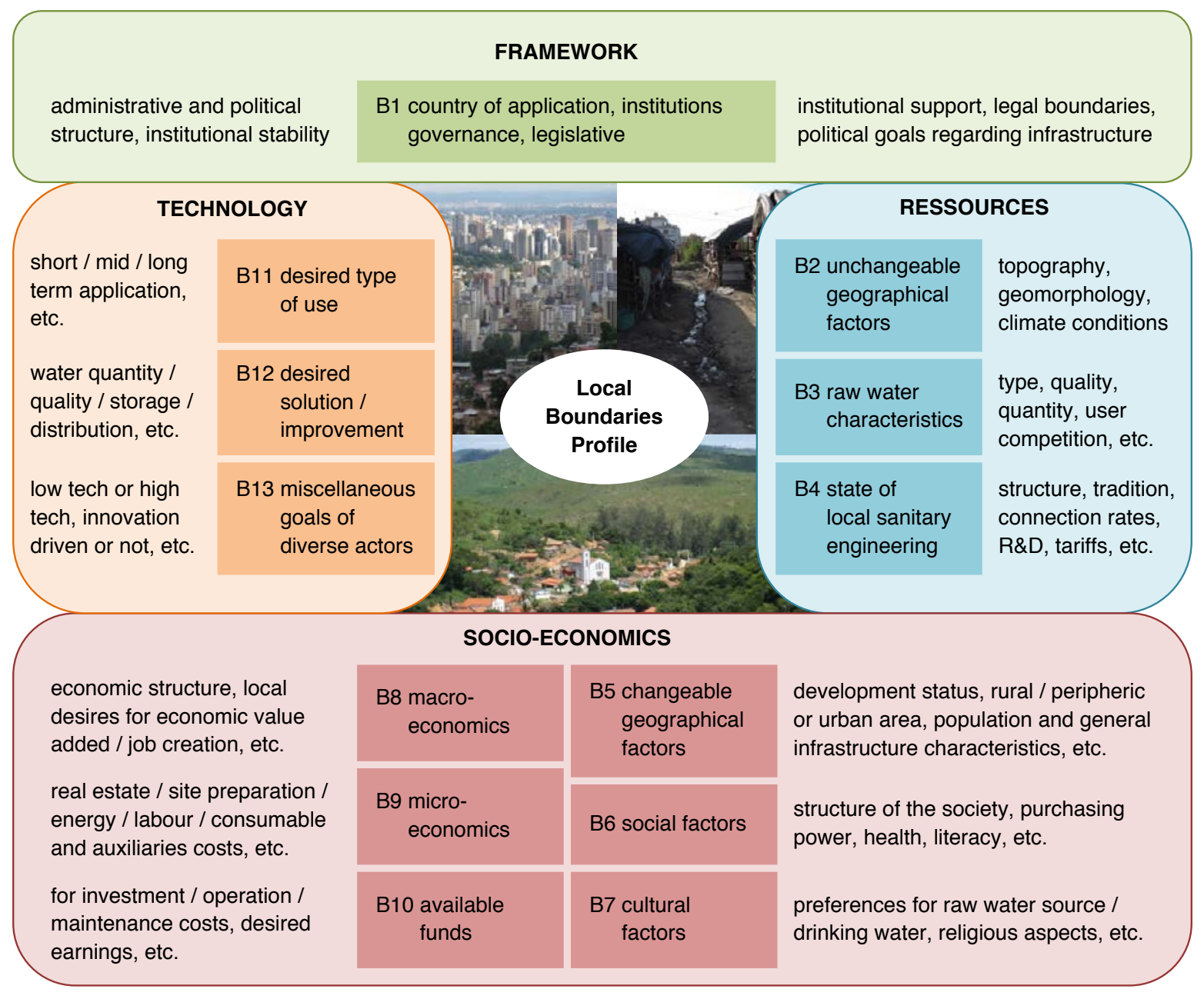

Figure 2: Open system of boundaries for decentralized water supply applications with examples of the 68 sub-boundary-groups 


\section{FRAMEWORK}

\begin{tabular}{|ll} 
A1 system type, \\
general plant \\
description
\end{tabular}

A2 tasks covered by the system

A3 plant operation strategy


\section{OPERATIONAL} BEHAVIOUR
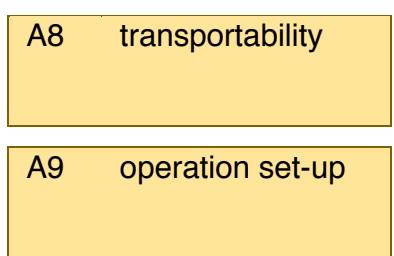

A10 movable character

\section{A11 plant behaviour} under continuing operation
SOCIO-ECONOMICS

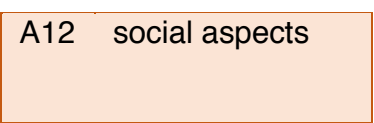

A13 macroeconomics

A14 microeconomics

A15 cost assessment

Figure 3: Structure and content units of the open system of attributes of decentralized water supply systems; further on divided into 64 sub-groups

\section{CONCEPT FOR RESILIENT AND FLEXIBLE DECENTRALIZED WATER INFRASTRUCTURE}

Decentralized water supply infrastructure incorporate many advantages compared to conventional centralized infrastructure systems, especially when it comes to resilience and flexibility. Nevertheless, the literature mentions associated disadvantages:

- Often a wide range of different systems is in use in a certain area,

- Difficulties with operation, maintenance and monitoring,

- Therefore, water quality and public health concerns.

To overcome these disadvantages the application of standardized, automated, SCADA equipped decentralized water treatment plants is suggested. Automation, quality measurement equipment and SCADA could improve quality transparency of decentralized water treatment plants. For the case that several similar plants are implemented, remote supervision from one control centre would be possible (look figure 4). This would allow centralized online control of the local plant status and of quality parameters. Water safety would be improved for the water customers. Supervision and O\&M would be facilitated for the water supplier. Regional O\&M centres could be responsible for regular O\&M. Irregular maintenance upon instruction from the central control centre could be executed from these regional O\&M centres as well. This concept is of special interest for

- rural regions where up to hundreds of decentralized water treatment plants need to be implemented and operated in small communities, i.e. e.g. for large surface states like Brazil.

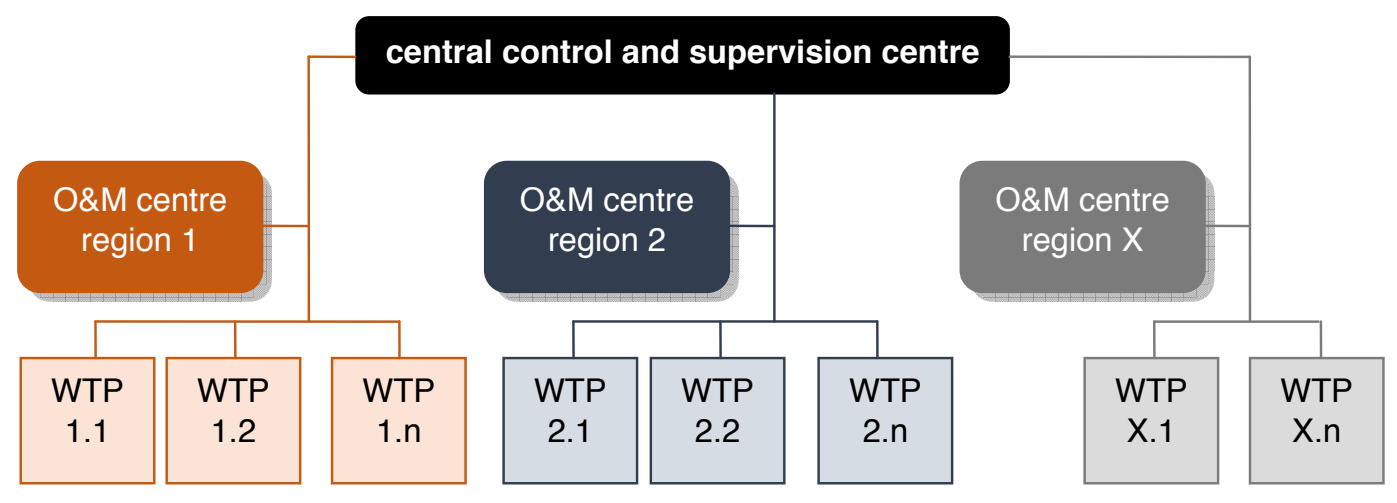

WTP... water treatment plant

Figure 4: Decentralized water supply supervision, operation and maintenance concept 


\section{PILOT PLANT TEST RESULTS AND OUTLOOK}

The backbone of the concept presented in figure 4 are the stand-alone water treatment plants. After compiling the local "Boundaries Profile" a related pilot plant was tested in the Brazilian State of Minas Gerais within the research project described in section three. The pilot plant was a Small Water System (look figure 1). All the equipment was fixed in two ten feet high cube containers which were electrically and hydraulically connected on site. A modular built up incorporated potential for future adaptability. The pilot plant's treatment steps were:

Hydrocyclone (alternatively) - Flocculation/coagulation and pH-value correction (alternatively) - Pre-oxidation (alternatively) - Drum filter or a plate separator - Aerator (alternatively) - Ultrafiltration - Reverse osmosis unit (alternatively) - Final chlorination and adding of fluorine (both alternatively).

Automation, equipment with online quality measurement devices and SCADA were the core features of pilot plant configuration and of its operation strategy. Personnel extensive, remote telecontrolled membrane water treatment was the desired goal. Results of in-depth on-site tests showed that both, the integrity of the treatment system under difficult raw water conditions as well as the compliance with the local drinking water quality provisions were guaranteed. However, regarding the supervision and O\&M concept the findings revealed general challenges under instable and extreme conditions:

- Telecommunication availability,

- Reliability of digital quality measurement equipment,

- Local supply chain management of hardware and consumables.

- Local understanding for the necessity of capacity building,

- The local personnel's education and knowledge level regarding advanced technology,

- Unclear expectations about what an automated membrane water treatment plant can and what it cannot. 